\title{
Motorized Circular Rail with RGB-D Sensor on Cart for Physical Rehabilitation
}

\author{
Ramón Panduro ${ }^{1}$, Lidia M. Belmonte ${ }^{1,2}$, Eva Segura ${ }^{2}$, Paulo Novais ${ }^{3}$, \\ José Pascual Molina ${ }^{1,4}$, Pascual González ${ }^{1,4}$, Antonio Fernández-Caballero ${ }^{1,2}$, \\ and Rafael Morales ${ }^{1,2}$ \\ 1 Universidad de Castilla-La Mancha \\ Instituto de Investigación en Informática de Albacete, 02071-Albacete, Spain \\ 2 Universidad de Castilla-La Mancha \\ Escuela Técnica Superior de Ingenieros Industriales, 02071-Albacete, Spain \\ 3 Universidade do Minho, Intelligent Systems Lab, Campus of Gualtar \\ 4710-057 Braga, Portugal \\ 4 Universidad de Castilla-La Mancha, Escuela Superior de Ingeniería Informática \\ 02071-Albacete, Spain
}

\begin{abstract}
This paper introduces a motorized circular rail managing the movement of two carts equipped with an RGB-D sensor each. The proposal is aimed at continuously tracking a person who is undergoing a series of physical rehabilitation exercises from two different viewpoints to monitor if the exercises are being correctly conducted. More concretely, this work offers all details of the trajectory calculation for safe movement of both carts on the motorized circular rail. Then, two study cases are presented to show the efficiency of the control algorithms implemented.
\end{abstract}

Keywords: Physical rehabilitation - Moving cart · Motorized circular rail.

\section{Introduction}

Assistance and rehabilitation systems based on computers have now become popular. These approaches typically use a depth camera that captures the figure of a human and transmits it to a processing node [1], [2]. Precisely, our research team has been working in providing RGB-D solutions for rehabilitation exercises (e.g. [3], [4]) in the last years. This kind of vision-based systems are based on human detection [5], [6] and tracking [7], [8], [9].

Nonetheless, it is mandatory to obtain good viewpoints from the tracked people who are exercizing themselves during physical rehabilitation programs. The RGB-D sensor has to be placed on the most convenient position in an intelligent manner to capture the most relevant body parts of the patient depending on the type of exercise. This is why, we are also developing mechanical solutions for moving RGB-D sensors during human rehabilitation exercises [10], [11].

This article describes the operation of a motorized circular rail capable of moving in an intelligent way a couple of carts equipped with an RGB-D camera 


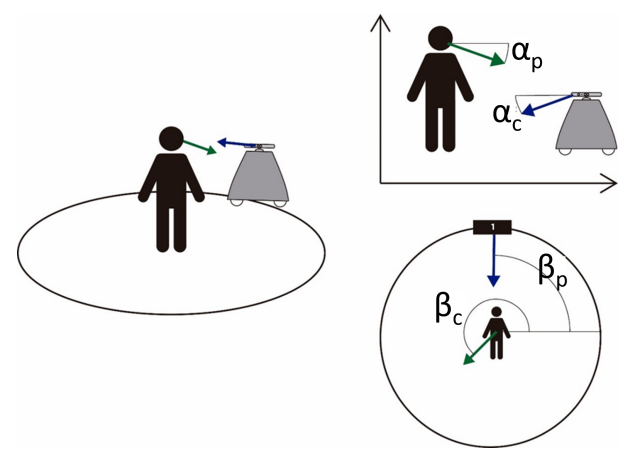

Fig. 1. Vector schemes.

each for monitoring the patient's body from two complementary views [12]. In this design, the patient is always in the center of the circle and the carts are arranged around him/her so as not to lose sight of the tracking vectors.

\section{Trajectory Calculation}

The control system of the motorized circular rail must calculate the trajectory of the two carts to place them in the ideal position to track the patient. The patient is introduced into the system as an input vector. It is assumed that both the patient's tracking vectors and the position vectors of the two carts are known in each instant. Several parameters are considered so that the trajectory is carried out correctly: the tilt and pan movements of the cameras (one per cart), the final angular position of the carts with respect to the rail, the vector that each cart must follow, and the possible collisions between both carts.

The formulas that govern the behavior of the carts are different depending on their master or slave role assigned. Thus, it is necessary to establish these roles according to the situation in which both carts are found. On the other hand, the angular position has to vary when a collision with the master cart is foreseen in the calculated trajectory of the slave. In this case, the slave must follow the opposite path.

The movement of the two carts (Q and S) is defined in relation to the tilt and the angular position. Since the patient is always in the center of the circular rail, the pan movement is not necessary and it is enough for the camera to remain straight. Therefore, in first place, the angular position that each cart must take is calculated. Then, the tilt movement that the RGB-D camera must make to focus on the desired patient's body area. Fig. 1 shows the nomenclature of angles and vectors that should be considered in the calculations.

In addition, a safety distance is added to ensure that a collision of the slave with the master cart will not occur. The maximum distance at which the two carts would collide in the most extreme case is calculated to obtain a safety zone. This corresponds to bot carts moving at full speed and slowing down with 


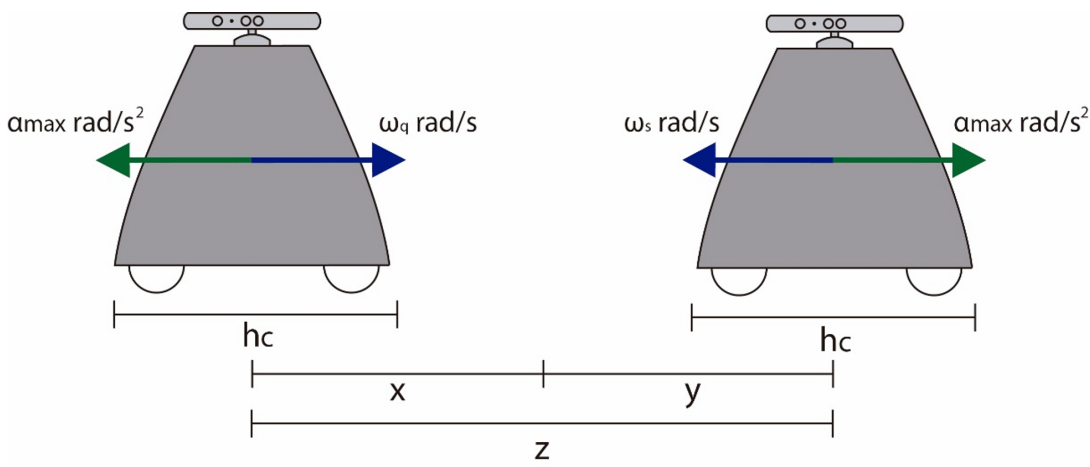

Fig. 2. Security distance of the carts.

maximum acceleration to reach zero speed. The maximums of speed and angular acceleration are $\alpha_{\max }=\pi / 4 \mathrm{rad} / \mathrm{s}^{2}$ and $\omega_{\max }=\pi / 2 \mathrm{rad} / \mathrm{s}$, respectively. The distance reached by a cart until it brakes is called $\theta$.

$$
0=\omega_{\max }^{2}-2 \cdot \alpha_{\max } \cdot \theta ; 0=\left(\frac{\pi}{2}\right)^{2}+\left(\frac{-2 \pi}{4}\right) \cdot \theta ; \theta=\frac{\pi}{2}
$$

The width $h_{c}$ of the carts is subtracted from the safety distance. Then, it is multiplied by a safety factor $\lambda_{s}$. Obviously, it would be interesting to estimate the safety distance according to the actual speed of both carts. Fig. 2 shows the scheme representing the safety distance considering the carts' angular velocities $\omega_{i}$, where

$$
x=\frac{\omega_{q}^{2}}{2 \alpha_{\max }} ; y=\frac{\omega_{s}^{2}}{2 \alpha_{\max }} ; z=\frac{\omega_{q}^{2}+\omega_{s}^{2}}{2 \alpha_{\max }}=\frac{\omega_{q}^{2}+\omega_{s}^{2}}{\pi / 2}
$$

The angle that covers the width of a cart, $\omega_{c}$, is defined by the following formula:

$$
\omega_{c}=\frac{h_{c}}{2 R}
$$

being $2 \omega_{c}$ the total safety angle for both carts. Subtracting this distance and multiplying by a safety factor $\lambda_{s}$, the final value of safety distance $z_{s}$ is gotten as

$$
z_{s}=\left(\frac{\omega_{q}^{2}+\omega_{s}^{2}}{2 \alpha_{\max }}-\frac{h_{c}}{R}\right) \cdot \lambda_{s}
$$

\section{$3 \quad$ Study Cases}

A couple of study cases are shown to validate the theoretical design of the tracking trajectories. Four different graphs are provided in both study cases. The first graph consists of a two-dimensional aerial view of the initial instant (starting positions of the carts). The second graph also uses an aerial view, but now showing the final positions of the carts after moving to track the patient. The other two 
graphs are used to check the tilt of the RGB-D camera. Two-dimensional views offer an initial and final graph of the cameras' vectors. A simplified tilt system is considered where a cart does not affect the position of its camera. Moreover, it is considered that the cameras are observing each other when their vectors have the same direction but alternate sense.

Master vs. slave cart role assignment is an important issue. This is why a complete analysis is carried out in both proposed study cases. Study case A shows a normal tracking situation whilst study case B bases on a possible collision situation. The numerical positioning data in both situations are shown in Table 1.

Table 1. Study data.

\begin{tabular}{ccc}
\hline Data (rad) & Study case A & Study case B \\
\hline$\beta_{P M}$ & $3 \pi / 4$ & $3 \pi / 4$ \\
$\beta_{P E}$ & 0 & $\pi$ \\
$\beta_{C Q}$ & $\pi / 2$ & $\pi / 2$ \\
$\beta_{C S}$ & $\pi / 4$ & $\pi / 4$ \\
$\alpha_{P M}$ & $\pi / 4$ & $\pi / 4$ \\
$\alpha_{P E}$ & $\pi / 7$ & $\pi / 7$ \\
$\alpha_{C Q}$ & $\pi / 3$ & $\pi / 3$ \\
$\alpha_{C S}$ & $-\pi / 5$ & $-\pi / 5$ \\
\hline
\end{tabular}

\subsection{Study Case A}

A tracking system has been tested with an immobile person in the centre of the rail. Thus, it is always possible to follow the patient's vectors (main and secondary) by keeping the camera pointing to the center. The values shown in Table 1 have been inserted as input values, generating the graphs illustrated in Fig. 3. The first graph shows the start moment, with cart Q depicted as a yellow vector and cart $\mathrm{S}$ in blue. The red vector symbolizes the main vector of the patient, while the green one is the secondary. The priority for the system is to choose the most efficient path to cover both vectors.

First of all, the roles that shortens the path taken by both carts as much as possible are defined for $\mathrm{Q}$ and $\mathrm{S}$. In case this distance is the same and there is no possibility of collision, cart Q takes the master role. In this particular study case $\mathrm{A}$, the system is in a normal situation, the shortest path calculation gives the role of master to cart $\mathrm{Q}$ and cart $\mathrm{S}$ is the slave. As shown in Fig. 3, the roles have been correctly assigned and the carts have moved to the expected final positions. In order to check if the system has selected the shortest path, Table 2 shows a comparison between the values calculated by the system and the selected final path. In both cases, the system selects the shortest path between the two possible paths, depending on whether clockwise or counterclockwise direction is chosen for carts Q and S. 

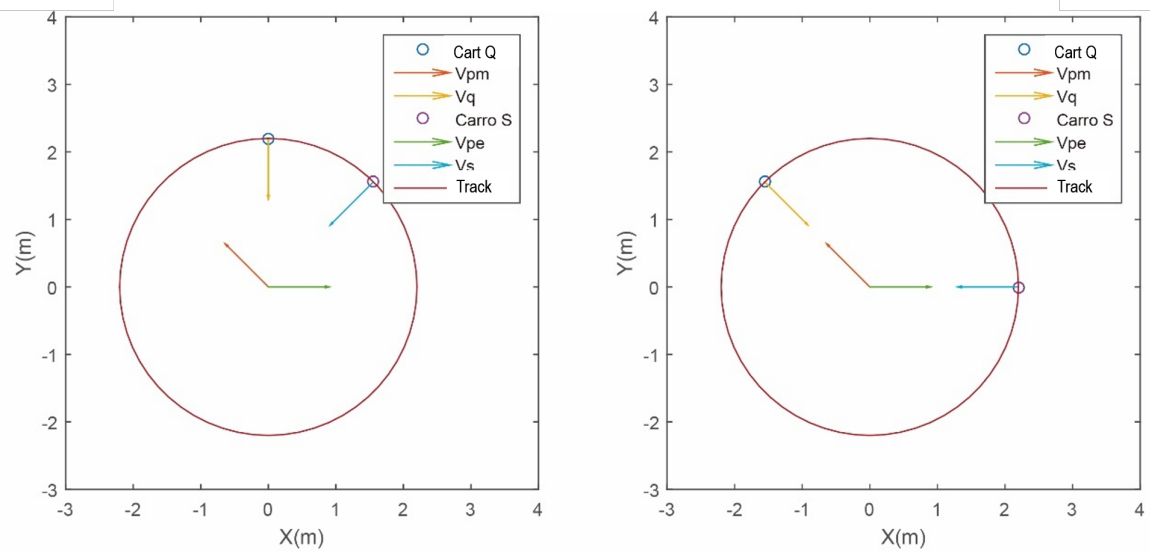

Fig. 3. Positioning graphs of study case A. Left: start positions. Right: final positions.

Table 2. Distance according to path.

\begin{tabular}{lrr}
\hline Data (rad) & Cart Q & Cart S \\
\hline Path clockwise & -5.4978 & -0.7854 \\
Path counterclockwise & 0.7854 & 5.4978 \\
Path selected & 0.7854 & -0.7854 \\
\hline
\end{tabular}

Secondly, it is interesting to check if the roles are correctly assigned so that the total distance traveled by both carts is minimal (see Table 3). Since cart Q is selected as master and $\mathrm{S}$ as slave, the system has correctly selected the shortest distance traveled by both carts.

Table 3. Distance according to role.

\begin{tabular}{lcc}
\hline Data $(\mathrm{rad})$ & $\mathrm{Q}$ master, S slave Q slave, S master \\
\hline Distance Q & 0.7854 & 1.5708 \\
Distance S & 0.7854 & 1.5708 \\
Distance total & 1.5708 & 3.1416 \\
\hline
\end{tabular}

The next issue to check is the tilt of the camera. After entering the data shown in Table 3, the graphs shown in Fig. 4 are obtained.

The camera has to tilt in order to point directly at the area that is to be observed as smallest path. As can be seen in the graph, the vector of cart Q is tilted until it is in the same direction as the patient's main vector, but in the opposite direction. In the same way, the vector of cart $\mathrm{S}$ is tilted to observe the patient's secondary vector. As with positioning, the system is able to select the shortest path (see Table 4). In this case, it is counterclockwise and clockwise for cart $\mathrm{Q}$ and cart $\mathrm{S}$, respectively. 

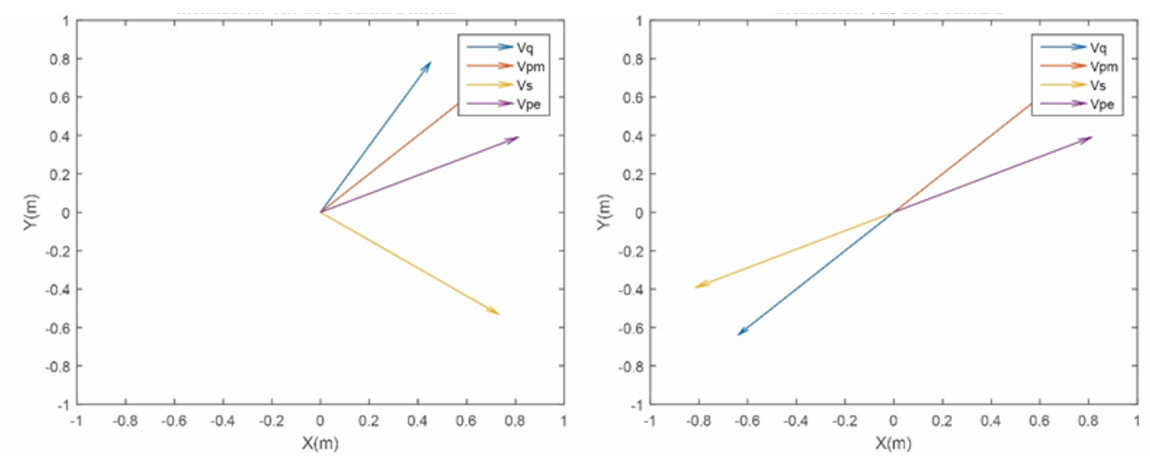

Fig. 4. Tilt graphs of study case A. Left: start positions. Right: final positions.

Table 4. Tilt according to path.

\begin{tabular}{lrr}
\hline Data (rad) & Cart Q & Cart S \\
\hline Path clockwise & -3.4034 & -2.0645 \\
Path counterclockwise & 2.8798 & 4.2187 \\
Path selected & 2.8798 & -2.0645 \\
\hline
\end{tabular}

The final displacement functions of carts $\mathrm{Q}$ and $\mathrm{S}$ are called $M_{C Q}$ and $M_{C S}$, respectively.

$$
M_{C Q}(\beta, \alpha)=(0.7854,2.8798) \operatorname{rad} ; M_{C S}(\beta, \alpha)=(-0.7854,-2.0654) \mathrm{rad}
$$

\subsection{Study Case B}

In this new study case, the position of $\beta_{P E}$ is modified to cause a collision possibility and thus to observe the system's reaction. It is checked how the change of roles affects the positioning. The graphs shown in Fig. 5 allow observing the evolution of the system with the new position of the patient's secondary vector.

Here, the roles are changed as consequence of the anticipation of a collision based on the calculation of the shortest distance. Collision means that one of the carts will interfere in the trajectory of the other. In this case, cart Q is placed as slave and $\mathrm{S}$ as master. This arrangement is initiated by introducing the camera data as input vectors. However, these data have been considered as the position of the carts to simplify the selection of roles. Next, the tilt of the cameras are evaluated as shown in Fig. 6.

As explained above, the choice of the tracking vectors is defined by the angular positions of the carts. In this case, the tilt has a different path than in study case A, even as they have to go to the same place as before. Here, it is cart $\mathrm{Q}$ that follows the patient's secondary vector, while the $\mathrm{S}$ cart tracks the patient's main vector. It can be seen in the graphs that the tracking task is done correctly. In this case, the movement functions of the carts are as follows:

$$
M_{C Q}(\beta, \alpha)=(1.5708,2.5432) \operatorname{rad} ; M_{C S}(\beta, \alpha)=(1.5708,-1.7279) \mathrm{rad}
$$



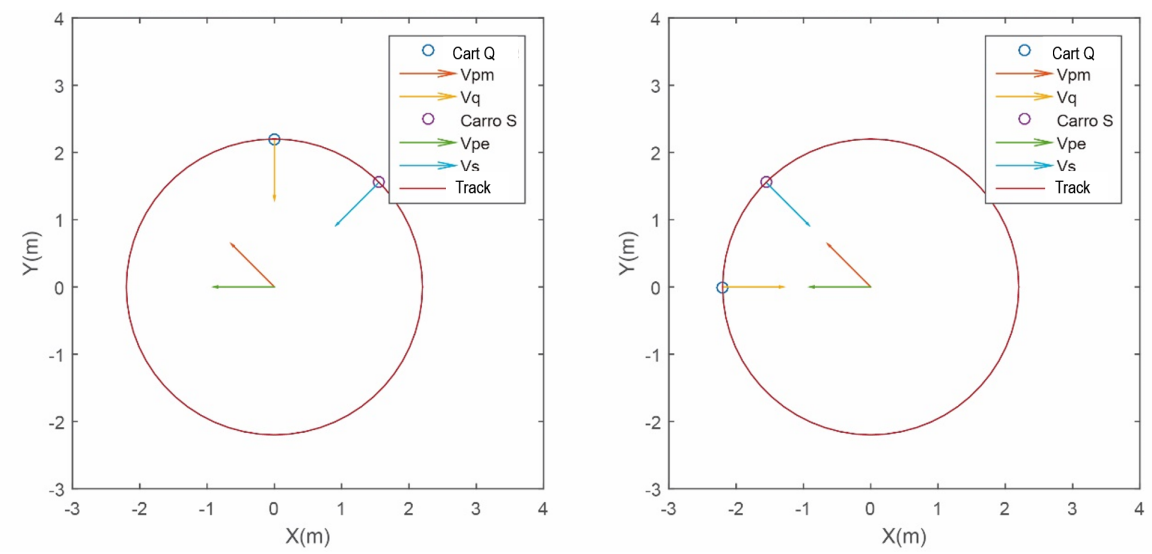

Fig. 5. Positioning graphs of study case B. Left: start positions. Right: final positions.
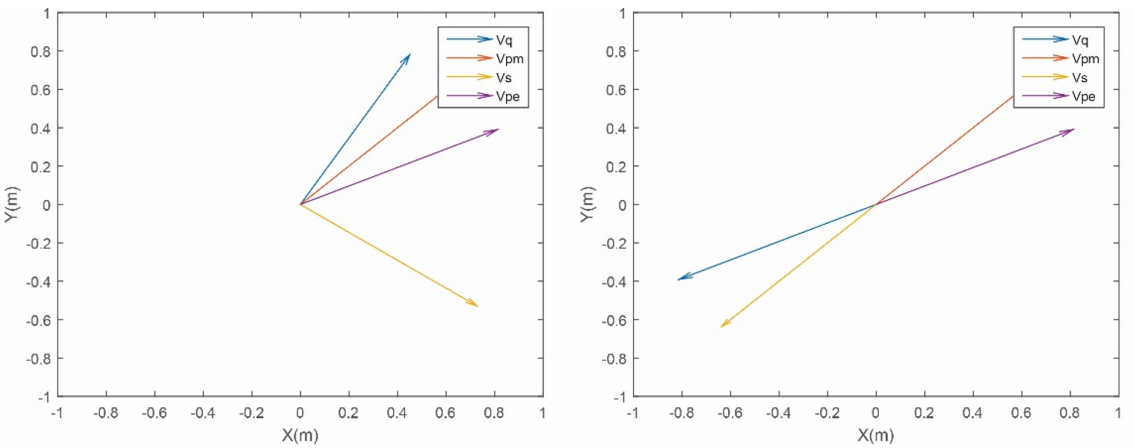

Fig. 6. Tilt graphs of study case B. Left: start positions. Right: final positions.

\section{Conclusions}

This paper has introduced a trajectory calculation algorithm for the movement of two carts (equipped with an RGB-D sensor) on a motorized circular rail. The final application of such couple of carts is continuous monitoring of a person who is fulfilling a series of physical rehabilitation exercises.

The paper has also described two study cases that show that the proposed algorithm possesses enough security to ensure that both carts will never collide during their tracking of the rehabilitation program of the human.

As future work, we propose: (a) to extend the trajectory planner to consider the free movement of the person within the working area of the proposed rail system; (b) to study different sensory strategies for conducting experimental trials that allow patients to test the system; (c) to conduct studies on users' perception for this kind of monitoring systems in order to draw conclusions about possible design improvements. 


\section{Acknowledgements}

This work was partially supported by Ministerio de Ciencia, Innovación y Universidades, Agencia Estatal de Investigación (AEI) / European Regional Development Fund (FEDER, UE) under DPI2016-80894-R and TIN2016-79100-R grants.

\section{References}

1. Chang, Y., Chen, S., Huang, J.: A Kinect-based system for physical rehabilitation: a pilot study for young adults with motor disabilities. Research in Developmental Disabilities, 32(6), 2566-2570 (2011)

2. Freitas, D., Gama, A. Da, Figueiredo, L., Chaves, T., Marques-Oliveira, D., Teichrieb, V., Araújo, C.: Development and evaluation of a Kinect based motor rehabilitation game. Simposio Brasileiro de Jogos e Entretenimento Digital, 144-153 (2012)

3. Oliver, M., Montero, F., Molina, J.P., González, P., Fernández-Caballero, A.: Multi-camera systems for rehabilitation therapies: a study of the precision of Microsoft Kinect sensors. Frontiers of Information Technology \& Electronic Engineering 17(4), 348-364 (2016)

4. Oliver, M., Montero, F., Fernández-Caballero, A., González, P., Molina, J.P.: RGB$\mathrm{D}$ assistive technologies for acquired brain injury: description and assessment of user experience. Expert Systems 32(3), 370-380 (2015)

5. Fernández-Caballero, A., Castillo, J.C., Serrano-Cuerda, J., Maldonado-Bascón, S.: Real-time human segmentation in infrared videos. Expert Systems with Applications 38(3), 2577-2584 (2011)

6. Fernández-Caballero, A., López, M.T., Saiz-Valverde, S.: Dynamic stereoscopic selective visual attention (DSSVA): Integrating motion and shape with depth in video segmentation. Expert Systems with Applications 34(2), 1394-1402 (2008)

7. Castillo, J.C., Fernández-Caballero, A., Serrano-Cuerda, J., López, M.T., Martínez-Rodrigo, A.: Smart environment architecture for robust people detection by infrared and visible video fusion. Journal of Ambient Intelligence and Humanized Computing 8(2), 223-237 (2017)

8. Fernández-Caballero, A., López, M.T., Serrano-Cuerda, J.: Thermal-infrared pedestrian ROI extraction through thermal and motion information fusion. Sensors 14(4), 6666-6676 (2014)

9. Moreno-Garcia, J., Rodriguez-Benitez, L., Fernández-Caballero, A., López, M.T.: Video sequence motion tracking by fuzzification techniques. Applied Soft Computing 10(1), 318-331 (2010)

10. Panduro, R., Oliver, M., Morales, R., González, P., Fernández-Caballero, A.: Motorized multi-camera slider for precise monitoring of physical rehabilitation. Lecture Notes in Computer Systems, 10070, 21-17 (2016)

11. Gascueña, J.M., Fernández-Caballero, A.: Agent-oriented modeling and development of a person-following mobile robot. Expert Systems with Applications 38(4), 4280-4290 (2011)

12. Mkhitaryan, A., Burschka, D.: RGB-D sensor data correction and enhancement by introduction of an additional RGB view. Proc. IEEE/RSJ Int. Conf. on Intelligent Robots and Systems, 1077-1083 (2013) 